\title{
Mileage-responsive Wind Power Smoothing
}

\author{
Lyu, Xue; Jia, Youwei; Xu, Zhao; Østergaard, Jacob
}

Published in:

IEEE Transactions on Industrial Electronics

Link to article, DOI:

10.1109/TIE.2019.2927188

Publication date:

2020

Document Version

Peer reviewed version

Link back to DTU Orbit

Citation (APA):

Lyu, X., Jia, Y., Xu, Z., \& Østergaard, J. (2020). Mileage-responsive Wind Power Smoothing. IEEE Transactions on Industrial Electronics, 67(6), 5209 - 5212. https://doi.org/10.1109/TIE.2019.2927188

\section{General rights}

Copyright and moral rights for the publications made accessible in the public portal are retained by the authors and/or other copyright owners and it is a condition of accessing publications that users recognise and abide by the legal requirements associated with these rights.

- Users may download and print one copy of any publication from the public portal for the purpose of private study or research.

- You may not further distribute the material or use it for any profit-making activity or commercial gain

- You may freely distribute the URL identifying the publication in the public portal

If you believe that this document breaches copyright please contact us providing details, and we will remove access to the work immediately and investigate your claim 


\title{
Mileage-responsive Wind Power Smoothing
}

\author{
Xue Lyu*, Student Member, IEEE, Youwei Jia*, Member, IEEE \\ Zhao Xu, Senior Member, IEEE, Jacob Østergaard, Senior Member, IEEE
}

\begin{abstract}
Conventional wind power smoothing control adopts hard-coded filtering algorithms to produce smoothed power output without considering the actual system need. This paper proposes a novel smoothing control paradigm in context of performance-based regulation service, in which the actual balancing need is considered and formulated as AGC regulation mileage. The proposed smoothing objective is to alleviate the regulation mileage while maximize wind energy harvesting. The effectiveness of the proposed framework is demonstrated through comparable case studies, through which the simulation results suggest a high potential for practical applications.
\end{abstract}

Index Terms-Wind energy, smoothing control, mileage, performance-based regulation service

\section{INTRODUCTION}

$\mathrm{T}$ ILL date, various wind power smoothing approaches have been reported whereas a general scientific question still remains open - to which extent such "smoothed" wind power profiles could be actually conducive to grid operation? To answer this question, this letter holds the perspective that wind power smoothing mechanism shall intrinsically correlate with main grid operation and address the system balancing need.

The state-of-the-art control methods (without integrating energy storage) for wind power smoothing generally rely on filtering-based algorithms (e.g. moving average (MA) [1], fuzzy-based discrete Kalman filter (KF) [2], ramp limits (RL) [3]) to produce smoothing command at the wind turbine (WT) level. Admittedly, grid frequency stability can be significantly crippled by instant generation-demand imbalance. Hence smoothing control should be essentially employed to alleviate

Manuscript received September 26; revised January 24, April 21; accepted June 7.

$X$. Lyu is with the Department of Electrical and Electronic Engineering, Southern University of Science and Technology, Shenzhen, China, and also with the Department of Electrical Engineering, The Hong Kong Polytechnic University, Hung Hom, Hong Kong. (email: xuelu111@gmail.com)

Y. Jia is with the Dept. of Electrical and Electronic Engineering, Southern University of Science and Technology, Shenzhen, China, jiayw@sustech.edu.cn)

Z. Xu is with the Dept. of Electrical Engineering, The Hong Kong Polytechnic University, Hung Hom, Hong Kong. (email: eezhaoxu@polyu.edu.hk).

J. Østergaard is with the Center for Electric and Energy, Department of Electrical Engineering, Technical University of Denmark, Kongens Lyngby 2800, Denmark. (E-mail: joe@elektro.dtu.dk).

*The authors are co-first authors. The first two authors contribute equally to this paper. system balancing pressure. Obviously, self-driven WT control is dependent solely on autogenous generation profiles yet are weakly correlated with grid frequency stabilization. Meanwhile, the mechanism of generating such control references lacks a consideration of WT operational constraints, thus the obtained references can be technically ineffective in terms of unreasonable over-/under-production. Most importantly, self-driven smoothing control may be economically inefficient since a significant amount of wind energy may be arbitrarily sacrificed through the predefined hard-coded smoothing algorithms.

This paper is dedicated to addressing the aforementioned drawbacks by incorporating wind power smoothing control with its consequent effects on system frequency regulation service cost. Towards this end, we newly develop a receding horizon control framework, which consists of an optimization module to generate optimal control reference by considering wind-fluctuation-induced frequency stabilization cost, and a real-time control module to timely track the smoothing reference. It is worth noting that the proposed framework is distinguished among most existing works in this field and holds significant merits as 1) smoothing references are obtained through system-perceived optimization; and 2) the effectiveness of WT over-/under-production at instant moments can be ensured through practical-constrained cascade control. Besides, we would like to emphasize that the computational efficiency of the formulated optimization is satisfactorily high.

\section{Problem Description}

In the performance-based regulation market, automatic generation control (AGC) units are engaged to provide frequency regulations with service charges, which covers regulation capacity payment and regulation mileage payment [4]. As to service providers, mileage payment implies the degree of frequency regulation engagement in the control period. On the other hand, a significant part of mileage payment relates to the randomness of wind energy. In this connection, under such a market environment, it would be much interesting and reasonable to control wind power fluctuations by considering WFs as active participants other than passive "free-runners". Analogous to mileage payment, we refer such fluctuation-induced service charge as mileage cost. In this sense, mileage cost quantitively amounts to the degree of wind power fluctuations for the system.

Smoothing out the wind power fluctuations actively in response to the actual system needs can have a positive impact on system frequency stabilization. In this paper, the smoothing task and AGC regulation are collectively formulated as a receding horizon optimization problem. This problem can be 
interpreted on two different levels. On the system level, the obtained optimal control references should satisfy minimizing the induced mileage cost and maximizing wind energy harvesting in a specified look-ahead time window. On the WT level, following the optimal control references necessitates effective control for over-/under-production in real time.

\section{Methodology}

To tack the abovementioned problem, a model predictive control framework is developed (as illustrated in Fig.1). Essentially, the overall performance relies on the synergy between the optimization module and WT control module.

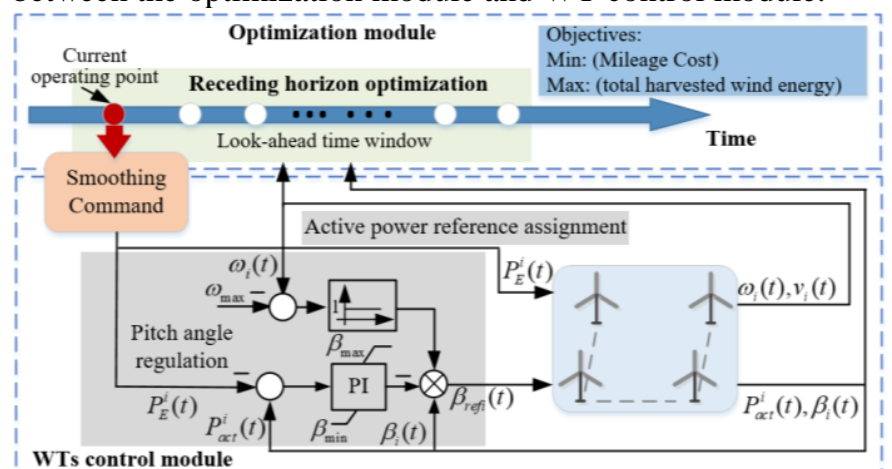

Fig. 1. The proposed mileage-responsive wind power smoothing control framework

\section{A. Receding Horizon Optimization}

The smoothing task is formulated as the following optimization problem in a specific look-ahead time window (i.e. from the present $t_{0}$ to a future time instance $t_{T}$ ), of which the decision variables are rotor speed $\omega_{i}(t)$ and pitch angle $\beta_{i}(t)$ of $i$-th WT at time $t$, and AGC regulation power $g_{k}(t)$ of unit $k$ at time $t$.

$$
\min -\alpha \sum_{t=t_{0}}^{t=t_{T}} \sum_{i=1}^{N} P_{E}^{i}(t) / P_{E}^{\max }+(1-\alpha) \sum_{t=t_{0}}^{t=t_{T}} \sum_{i=1}^{N}\left(\gamma_{t} g_{i}(t)\right)^{2} /\left(C_{\text {mileage }}^{\max }\right)^{2}
$$

s.t.

$$
\begin{gathered}
0 \leq P_{E i}^{t} \leq P_{M P P i}^{t}+P_{K i} \\
\omega_{\min } \leq \omega_{i}(t) \leq \omega_{\max } \\
\beta_{\min } \leq \beta_{i}(t) \leq \beta_{\max } \\
P_{\text {scheduled }}^{t}+P_{E}(t)+\sum_{i=1}^{n} g_{i}(t)=P_{\text {load }}^{t} \\
-g_{\text {cap }}^{k} \leq g_{k}(t) \leq g_{\text {cap }}^{k}
\end{gathered}
$$

var

$\omega_{i}(t), \beta_{i}(t) \in \mathbb{R}^{+}, g_{k}(t) \in \mathbb{R}, t \in\left[t_{0}, t_{T}\right], i \in[1, N], k \in[1, n]$.

In the objective function $(1), P_{E}^{i}(t)$ is the electric power output of the $i$-th WT at time slot $t$, which can be expressed as:

$$
P_{E}^{i}(t)=0.5 \rho A v_{t}^{3} C_{P}^{i}(t)-J \omega_{i}(t)\left[\omega_{i}(t)-\omega_{i}(t-1)\right] / \Delta t
$$

where $\rho$ is air density; $A$ is rotor swept area; $v_{t}$ is wind speed; $C_{P}$ is power coefficient; $J$ is the equivalent moment of inertia of the turbine blades and generator. In modelling the total amount of wind energy harvesting, $C_{P}$ normally has highly non-linear relationship with rotor speed and pitch angle. For simplicity, we adopt the following polynomial regression [5] that gives rise to satisfactorily good fitting precision (i.e. fitting error follows
MAPE $=0.1236$, RMSE $=0.1112$ ).

$C_{P i}^{t}=\left[c_{11} \beta_{i}(t)^{2}+c_{12} \beta_{i}(t)+c_{13}\right] \lambda_{i}(t)^{2}+\left[c_{21} \beta_{i}(t)^{2}+c_{22} \beta_{i}(t)+c_{23}\right] \lambda_{i}(t)$

$+\left[c_{31} \beta_{i}(t)^{2}+c_{32} \beta_{i}(t)+c_{33}\right]$

where $c_{i j}$ is regression parameter; $\lambda_{i}(t)=\omega_{i}(t) R / \beta_{i}(t)$ is tip speed ratio; $R$ is the turning radius of a WT.

The second term of the objective function (1) represents the mileage cost, where $\gamma_{t}$ is mileage-based clearing price in regulation markets at time $t ; P_{E}^{m a x}$ and $C_{\text {mileage }}^{\text {max }}$ are the maximum values of wind farm power output and mileage cost, respectively.

The objective function (1) integrates two independent normalized objectives (i.e. maximizing wind energy harvesting and minimizing wind fluctuation induced mileage cost) through the weighted-sum strategy. The trade-off of these two objectives can be controlled by the weighting coefficient $\alpha$. Under different operating conditions, specific rationales of selecting proper weights can be adopted. For example,

- Under normal conditions, given that the preferences of maximizing wind power output and minimizing mileage cost are unbiased, the weight $\alpha$ can be assigned as 0.5 to represent the equal importance of the objectives.

- Under abnormal conditions, e.g. system contingency occurs, a large weight $\alpha$ can be assigned to alleviate AGC regulation burden. In this way, the optimal solution trends to smooth out the power fluctuations of the wind farms. Naturally, larger $\alpha$ can result in more smoothened wind power output. The determination of $\alpha$ should be associated with the level of AGC regulation pressure and relevant reserve.

In this paper, to demonstrate the effectiveness of the proposed control paradigm in a general way, the Pareto optimality is presented in Section IV, where one example case, i.e. $\alpha=0.5$ representing unbiased preferences is presented in detail.

In quantifying the mileage cost, the clearing price $\gamma_{t}$ is typically determined by ISO based on specific market information, which covers bid-in regulation capacity, mileage price, and historical performance indices of AGC units [4]. Detailed price-clearing mechanisms are essential yet beyond the scope of the current work, hence will not be discussed further in this paper. Interested readers can refer to relevant literatures (e.g. [4]).

The formulated optimization is subject to several constraints. Constraints (2a)-(2c) capture the constraints of maximal WT electric power output, rotor speed and pitch angle, respectively, where $P_{M P P}^{t}$ is the captured mechanical power through the maximum power point tracking (MPPT) algorithm; $P_{K}$ is the maximum electric power that can be converted from the kinetic energy stored in the rotational system, which is expressed as,

$$
P_{K}^{i}(t)=0.5 J\left[\omega_{i}(t-1)^{2}-\omega_{i}^{M P P}(t)^{2}\right] / \Delta t
$$

where $\omega_{i}^{M P P}(t)$ is rotor speed of $i$-th WT under MPPT status at time $t$.

Constraint (2d) ensures that the instant net load can be satisfied by the scheduled power generation $P_{\text {scheeduled }}^{t}$ and AGC regulation. Constraint (2e) captures the maximal change of AGC regulation power, where $g_{\text {cap }}^{i}$ is the regulation capacity of 


\section{AGC unit $i$.}

\section{B. Wind Turbines Cascade Control}

WT output power needs to be controlled to track the smoothing power commands obtained by the optimization module. In this paper, a cascade strategy is proposed to realize the smoothing control objective. As illustrated in Fig.1, the proposed control strategy is not dependent on accessional energy storage system. It takes full advantage of the WT self-capability by sequentially utilizing rotor speed control and pitching control to meet the control command.

Echoed with the first optimization objective of maximizing wind energy harvesting, rotor speed control would preferentially be utilized to achieve over-/under-production by releasing/storing excessive kinetic energy from the rotating rotor. This part of controller features high energy utilization efficiency in managing redundant or insufficient wind energy through WTs. Nevertheless, the active power support provided via rotor speed control is subject to strict WT physical constraints as expressed in (2a). As shown in Fig.1, in case that rotational speed exceeds its upper limit (i.e. $\omega_{i} \geq \omega_{\max }$ ), pitching control will be activated to offer further power curtailment capability. Pitching is at the lowest priority in the proposed strategy so as to reduce the wastage of wind energy and mitigate the fatigue of WTs.

\section{CAse Studies}

The proposed method is preliminarily tested on a simple-yet-typical operation scenario as shown in Fig.2. In this testing system, AGC units complement the power imbalance between scheduled energy and net load. The AGC regulation mileage is represented as the shaded areas. Smoothing out wind power fluctuations to address the actual system needs amounts to reducing the shaded area in real time. The simulation parameters are predefined in Table I. The price clearing mechanism can be referred to [5].
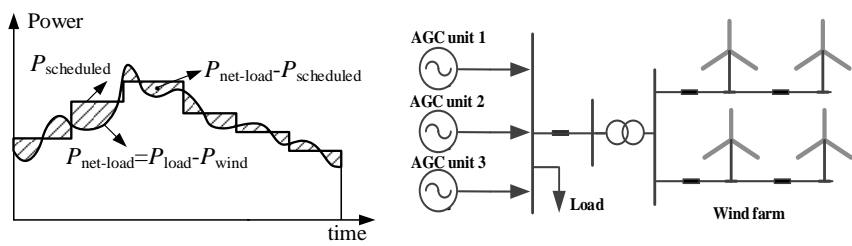

Fig. 2. Testing system

Table I

System Parameters

\begin{tabular}{|c|c|}
\hline \multirow{4}{*}{ AGC } & $\begin{array}{l}p_{m}: \text { normalized regulation performance score } \\
P_{o f f e r}^{i}: \text { mileage bidding price of unit } i(\$ / \mathrm{MW}) \\
g_{\text {cap }}^{i}: \text { maximum biddable capacity of unit } i(\mathrm{MW})\end{array}$ \\
\hline & $p_{m}^{1}=0.7168 \quad P_{o f f e r}^{1}=2 \$ / M W \quad g_{\text {cap }}^{1}=1.5$ \\
\hline & $p_{m}^{2}=0.6074 \quad P_{\text {offer }}^{2}=4 \$ / M W \quad g_{\text {cap }}^{2}=4$ \\
\hline & $p_{m}^{3}=1 \quad P_{\text {offer }}^{3}=1 \$ / M W \quad g_{\text {cap }}^{3}=2.5$ \\
\hline $\begin{array}{l}\text { Wind speed } \\
\text { data }\end{array}$ & Online accessible [6] \\
\hline Wind farm & $\begin{array}{l}\text { The studied wind farm consists of } 4 \text { NREL 5MW DFIG } \\
\text { wind turbines with total } 20 \mathrm{MW} \text { generation capacity. }\end{array}$ \\
\hline
\end{tabular}

In this case study, a typical time series of wind speed data for 1 hour is considered. The formulated non-linear optimization problem is solved every 4 seconds to supdate the power command, which is consistent with the AGC control cycle. The lookahead time window is set to be 20 s, during which the wind speed prediction accuracy can be satisfactorily high (as referred to [7], prediction performance for 4-s wind speed is acceptably accurate, i.e. $\mathrm{RMSE}=0.9435$, MAPE $=0.0603$ ). In our study, the optimization part can be readily solved in 0.2732 s (this computation time is averaged by 10000 individual simulations based on Dell Precision Tower with CPU E5-2650 v4@2.20GHz (2 processors)).

As discussed in Section III, the optimal smoothing references can be adjusted by the weighting coefficients, thus result in different smoothing effects to satisfy corresponding operation conditions. In this paper, one example case (i.e. $\alpha=0.5$ presenting unbiased preference for the two objectives) is shown in Fig.4. By changing $\alpha$ in a feasible region, the Pareto front is obtained and shown in Fig. 3. It is obvious that the increase of $\alpha$ can simultaneously lead to the increase of total generation output and mileage cost. In practical application in industry, decision-makings from system operators should compromise the total wind power production and its fluctuation-induced mileage cost under different operation conditions.

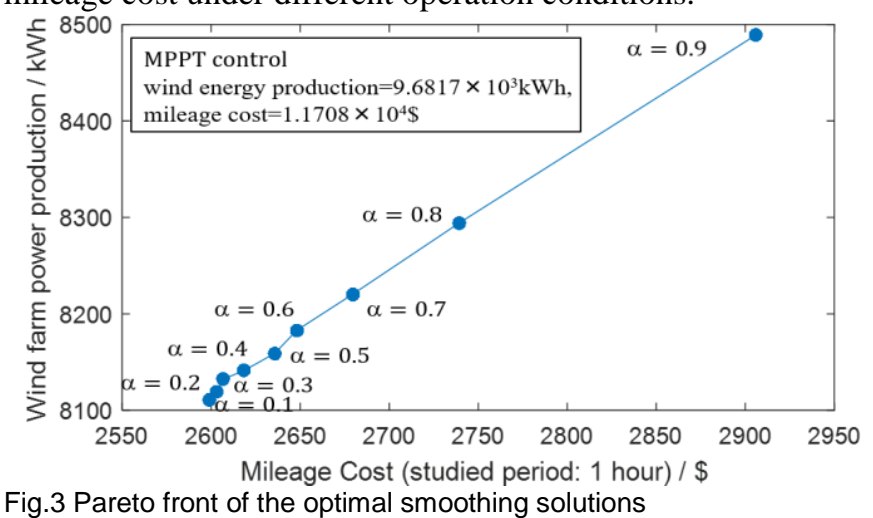

According to the smoothing objective (1) concerned in this paper, we define $f$ (in Table II) as a dimensionless index to quantify the smoothing effect in terms of total wind power output and mileage cost, which represents the tradeoff between the two objectives. Comparisons between the proposed method and the state-of-the-art techniques are reported in Table II. In general, filtering-based methods yield deterministic smoothing results, which cannot adapt to the changing system operational status. Overall, the proposed method generates the output profiles with minimum $f$.

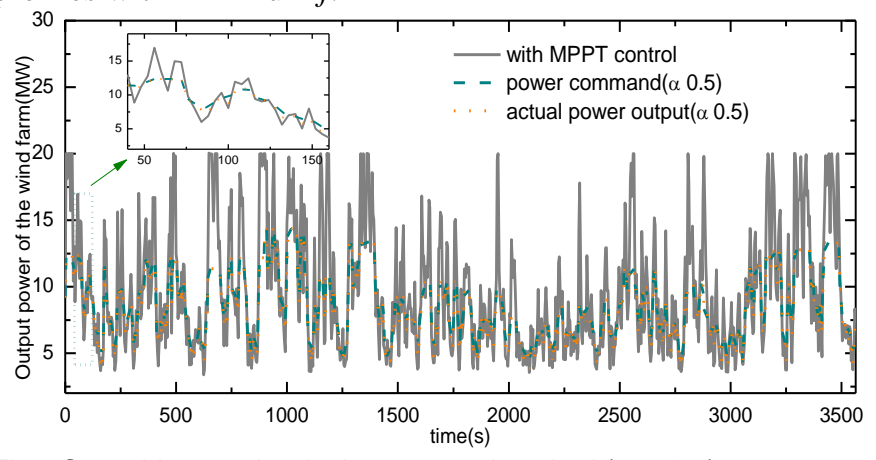

Fig.4 Smoothing results via the proposed method $(\alpha=0.5)$

Table II

COMPARISON RESULTS ON SMOOTHING PERFORMANCE

Smoothing $\quad f=\sum_{t=1}^{T}\left[-\alpha P_{E}(t) / P_{E}^{\max }+(1-\alpha)\left(\gamma_{t} g_{i}(t)\right)^{2} /\left(C_{\text {mileage }}^{\max }\right)^{2}\right] / T$ 


\begin{tabular}{c|c|c|c|c|c}
\hline \hline $\begin{array}{c}\text { index } \\
\text { (dimensionless) }\end{array}$ & $\alpha=0.1$ & $\alpha=0.3$ & $\alpha=0.5$ & $\alpha=0.7$ & $\alpha=0.9$ \\
\hline $\begin{array}{c}\text { Proposed } \\
\text { method }\end{array}$ & 4.7048 & 4.0584 & 2.8510 & 1.6068 & 0.2690 \\
\hline MA & 5.7965 & 4.4169 & 3.0374 & 1.6578 & 0.2783 \\
\hline KF & 6.3447 & 4.8418 & 3.3389 & 1.8360 & 0.3331 \\
\hline RL & 5.9476 & 4.5342 & 3.1209 & 1.7075 & 0.2942 \\
\hline \hline
\end{tabular}

\section{CONCLUSION}

This paper newly proposes an optimal wind power smoothing control framework, which is successfully tested through a preliminary case study. It is highlighted that the proposed system-oriented smoothing control is advantageous over conventional hard-coded self-driven algorithms to ensure genuinely smoothed operation of WTs subject to various constraints. Future work is underway to further enhance the proposed smoothing framework by considering wake interactions among turbines.

\section{REFERENCES}

[1] X. Lyu, J. Zhao, Y. Jia, Z. Xu, and K. P. Wong, "Coordinated control strategies of PMSG-based wind turbine for smoothing power fluctuations," IEEE Transactions on Power Systems, vol. 34, no. 1, pp. 391-401, 2019.

[2] D. Lamsal, V. Sreeram, Y. Mishra, and D. Kumar, "Smoothing control strategy of wind and photovoltaic output power fluctuation by considering the state of health of battery energy storage system," IET Renewable Power Generation, vol. 13, no. 4, pp. 578-586, 2019.

[3] Y. Zhou, Z. Yan, and N. Li, "A novel state of charge feedback strategy in wind power smoothing based on short-term forecast and scenario analysis," IEEE Transactions on Sustainable Energy, vol. 8, no. 2, pp. 870-879, 2017.

[4] A. Sadeghi-Mobarakeh and H. Mohsenian-Rad, "Optimal bidding in performance-based regulation markets: An mpec analysis with system dynamics," IEEE Transactions on Power Systems, vol. 32, no. 2, pp. 1282-1292, 2017.

[5] H. Ye, W. Pei, and Z. Qi, "Analytical modeling of inertial and droop responses from a wind farm for short-term frequency regulation in power systems," IEEE Transactions on Power Systems, vol. 31, no. 5, pp. 3414-3423, 2016.

[6] Online available: $\mathrm{http}: / / \mathrm{www} . w i n d d a t a . c o m / /$

[7] X. Luo et al., "Short-term wind speed forecasting via stacked extreme learning machine with generalized correntropy," IEEE Transactions on Industrial Informatics, vol. 14, no. 11, pp. 4963-4971, 2018. 